\title{
On the Relations between Eigenvalues and Eigenvectors for Matrices Resulting from Pre and Post Multiplication by the Transpose
}

\author{
By V. J. Law and R. H. Fariss
}

Abstract. Relationships between the eigenproblem associated with the matrices $A^{T} A$ and $A A^{T}$ are derived. If the larger problem is solved first, then the eigenvalues and eigenvectors associated with the smaller problem may easily be computed from the derived relationships.

Consider a nonsquare matrix $A$ of $\operatorname{rank} m$ and of dimension $c \times d$. The product $A^{T} A$ is symmetric and positive semidefinite, and hence possesses an eigenvectoreigenvalue relationship of the form

$$
S^{T}\left(A^{T} A\right) S=D,
$$

where $S$ is a normal orthogonal matrix containing eigenvectors as columns, and $D$ is a diagonal matrix containing eigenvalues as the diagonal elements. $S$ and $D$, both of dimension $d \times d$, can be ordered so that any zero eigenvalues, resulting from $A^{T} A$ having excess dimension over the rank $m$, are at the bottom:

$$
D=\left[\begin{array}{c:c}
E & 0 \\
\hdashline & \\
\hdashline 0 & 0
\end{array}\right]
$$

where $E$ is an $m \times m$ submatrix with nonzero diagonal elements. Correspondingly, the ordered $S$ can be partitioned as

$$
S=\left[S_{m}: S_{p}\right]
$$

so that the left-hand part $S_{m}$ contains those eigenvectors associated with nonzero eigenvalues. Here $p=d-m$.

The positive semidefinite property of $A^{T} A$ assures that the diagonal elements of $E$ are positive, hence $E$ can be "square-rooted":

$$
E=E^{1 / 2} E^{1 / 2}
$$

with $E^{1 / 2}$ being a diagonal matrix containing the square roots of the nonzero eigenvalues of $A^{T} A$.

The basic relationship (1) can be modified by premultiplication by $S$ to give

$$
\left(A^{T} A\right) S=S D \text {. }
$$

If (5) is written in partitioned form, the following result becomes obviously valid:

$$
A^{T} A S_{m}=S_{m} E
$$

Received December 11, 1967. 
A normal orthogonal vector set can be constructed as follows: First substitute (4) into (6) and premultiply by $S_{m}{ }^{T}$, then premultiply and postmultiply by $E^{-1 / 2}$ to give

$$
\left(A S_{m} E^{-1 / 2}\right)^{T}\left(A S_{m} E^{-1 / 2}\right)=I .
$$

Equation (6) is a good starting point for investigating the eigenproblem of $A A^{T}$. Premultiplying (6) by $A$ and postmultiplying that result by $E^{-1 / 2}$ gives

$$
\left(A A^{T}\right)\left(A S_{m} E^{-1 / 2}\right)=A S_{m} E^{1 / 2}=\left(A S_{m} E^{-1 / 2}\right) E .
$$

Now, from (7) and (8) it follows that

$$
\left(A S_{m} E^{-1 / 2}\right)^{T}\left(A A^{T}\right)\left(A S_{m} E^{-1 / 2}\right)=E .
$$

Now denote the eigenvector-eigenvalue relationship for $A A^{T}$ similarly to (1) as

$$
R^{T}\left(A A^{T}\right) R=D^{*},
$$

where $R$ and $D^{*}$ are of dimension $c \times c$. Reorder $R$ and $D^{*}$ to obtain

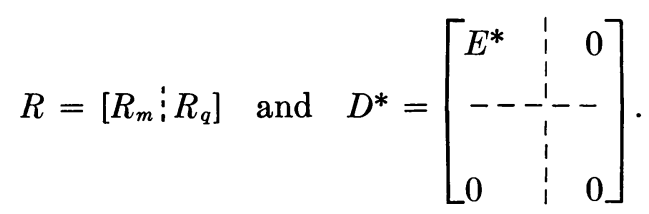

Here $q=c-m$ and $E^{*}$ is an $m \times m$ diagonal submatrix.

The following properties are well known:

(1) $E^{*}=E$.

(2) $R_{q}$ is arbitrary, subject to the restrictions $R_{q}{ }^{T} R_{m}=0, R_{q}{ }^{T} R_{q}=I$.

The important result of the paper is that

$$
R_{m}=A S_{m} E^{-1 / 2}
$$

which follows from an examination of (9).

In conclusion, if the larger problem (i.e., the eigenproblem associated with $A^{T} A$ ) is solved first, then the complete eigenproblem associated with $A A^{T}$ may be computed by using the above-mentioned well-known properties together with the result (11).

Tulane University

New Orleans, Louisiana 70118

Monsanto Company

Springfield, Massachusetts 01101 